\title{
The 68C Glue Puff of Drosophila
}

\author{
E.M. Meyerowitz, M.A. Crosby, M.D. Garfinkel, C.H. Martin, P.H. Mathers, \\ AND K. VIJAYRAGHAVAN \\ Division of Biology, California Institute of Technology, Pasadena, California 91125
}

\begin{abstract}
Drosophila melanogaster begins its life as a fertilized egg, which undergoes embryogenesis within an egg shell for about a day and then hatches from the egg as a first instar larva. The wormlike larva eats yeast for a day, then sheds its first instar skin and crawls out as a second instar larva. One day later another molt occurs, resulting in a third instar larva. The third instar stage lasts approximately 40 hours at $25^{\circ} \mathrm{C}$; toward the end of this stage the larva crawls out of its medium and onto a dry surface. After a few hours of wandering, it stops, contracts, and secretes a protein glue from its salivary glands that hardens and attaches the larva to its substrate (Fraenkel and Brookes 1953). The affixed larva then tans its third larval instar cuticle into a puparial case, and some hours later pupates within this case. Complete metamorphosis follows pupation, and after several days the pupal case is opened and a new adult emerges.
\end{abstract}

The third instar salivary glands that are the source of the secreted glue consist of a pair of separate lobes connected by a common anterior duct. Each lobe is a blind sac with a simple lumen; the lumen communicates with the anterior duct, and through the duct with the larval mouth, and the outside world. Each lobe contains approximately 130 giant cells, which were set aside during embryogenesis, and have enlarged without cell division since that stage. The enlargement of the cells parallels an increase in the DNA content of each cell: As the gland develops the chromosomes of its cells replicate up to 11 times, with the replicated chromatids staying aligned in register, to form the familiar polytene chromosomes that exist in many dipteran tissues (Berendes and Ashburner 1978). These chromosomes are enormous, with a diameter of around $5 \mu \mathrm{m}$, and lengths of hundreds of micrometers. They show an abundance of cytological detail. One prominent cytological feature is the pattern of bands: Bands are darkstaining transverse stripes on the chromosomes that sit in a constant relation to the genetic map, and thus to the DNA sequence. They are unchanging features, with the same banding pattern recognizable in all stages of salivary gland development in which the chromosomes are large enough to allow the banding pattern to be resolved. Individual bands can be identified, and each is named according to a standard system (Bridges 1935). In addition to bands, polytene chromosomes have puffs, transient structures which are local regions of chromatin decondensation visible in the light microscope as areas of expanded diameter and indistinct banding. Puffs are normally sites of highly active tran- scription (Ashburner and Berendes 1978). The puffs seen in midthird instar larvae, when the salivary chromosome banding pattern is first distinct enough to allow individual puff locations to be ascertained, are called the intermolt puffs. There are about 10 of them, with the most prominent found in band region $3 \mathrm{C}$ on the $\mathrm{X}$ chromosome, 25B on the left arm of the second chromosome, $68 \mathrm{C}$ on the left arm of the third chromosome, and $90 \mathrm{BC}$ on the right arm of the third chromosome. About five hours before puparium formation these puffs regress, with restoration of the normal diameter and sharp banding pattern of the previously puffed regions. Simultaneous with the regression of the intermolt puffs is the appearance of a new set of puffs called the early puffs; these regress by the time of puparium formation, and are followed by a set of puffs called the late puffs. The changes in puffing pattern reflect changing patterns of gene expression in the developing salivary glands (Ashburner 1967, 1973).

The best characterized set of puffs is the intermolt set. Both genetic and molecular experiments have shown that these puffs contain the structural genes for the polypeptides that comprise the salivary gland glue (Korge 1975, 1977; Akam et al. 1978; Muskavitch and Hogness 1980; Velissariou and Ashburner 1980, 1981; Crowley et al. 1983; Gautam 1983; Guild and Shore 1984). This glue consists of at least eight polypeptides that start to be synthesized in the salivary glands in early to midthird instar, and stop synthesis at the time of puparium formation (Beckendorf and Kafatos 1976). The signals that induce these puffs are not known; they regress in response to a steroid hormone, ecdysterone. This response occurs both in vivo, as a result of release of the hormone from the larval ring gland, and in vitro, when midthird instar larval salivary glands are cultured in a medium containing ecdysterone (Ashburner 1973). If protein synthesis inhibitors are added to cultured intermolt salivary glands along with ecdysterone, the regression of at least three of the intermolt puffs (3C, 25B, and 68C) still occurs, indicating that the regression is a direct response to the hormone, and does not require intermediate steps of gene expression (Ashburner 1974).

The intermolt puffs thus represent a set of developmentally and hormonally controlled genes that express in a tissue-specific manner and code for known products of known function. In addition, their expression is accompanied by visible and striking changes in chromatin structure. To understand better the mechanisms of tissue-specific and developmentally specific gene 
expression, and the relation of puffing to gene expression, we are pursuing molecular and genetic studies of the $68 \mathrm{C}$ puff.

\section{DNA Sequence Organization of the 68C Puff}

The cloning of the $68 \mathrm{C}$ puff DNA started with isolation of cDNA clones representing RNAs specific to and abundant in midthird instar salivary glands. These were screened by in situ hybridization to salivary gland polytene chromosomes and five major classes of $\mathrm{CDNA}$ inserts were found. Clones within each class cross-hybridize, clones in different classes do not. One class hybridizes to the $3 \mathrm{C}$ glue puff (which codes for the sgs4 glue polypeptide) in in situ hybridization experiments, and one to the $90 \mathrm{BC}$ glue puff (the site of the structural gene of the sgs-5 glue protein). The remaining three classes of clones hybridize in situ to $68 \mathrm{C}$ (Wolfner 1980). Each of the three 68C cDNA clone classes hybridizes to a different third instar salivary gland RNA; the three RNAs are 360, 320, and 1120 nucleotides in length. The RNAs are all polyadenylated and polysomal (Meyerowitz and Hogness 1982). By comparing conceptual translations of the cDNA sequences with partial amino acid sequences of different glue polypeptides, it was found that each of the $68 \mathrm{C}$ RNAs codes for a different glue component (Crowley et al. 1983). The 1120-nucleotide RNA is the mRNA for the sgs-3 glue polypeptide, which had previously been shown by genetic mapping to be coded in or near the 68C puff (Korge 1975; Akam et al. 1978). The other two RNAs coded for two previously undescribed glue fractions: the 320-base RNA is the mRNA for the sgs7 polypeptide, and the 360 -base RNA is for the sgs-8 polypeptide (Crowley et al. 1983).

The amino acid sequences of the three $68 \mathrm{C}$ glue proteins are related: All three have a 23-residue aminoterminal signal peptide that is cleaved off before secretion of the mature protein, and all of the proteins have carboxyterminal regions of about 50 amino acids that show considerable homology with each other. In addi- tion to these two modules, the sgs- 3 protein has a third, central, module that is 234 amino acids long and largely comprised of tandem repeats of the five amino acids Pro-Thr-Thr-Thr-Lys. Thus, the secreted sgs-7 and sgs8 peptides are small and similar to each other, whereas the sgs-3 protein is much larger, and contains over $40 \%$ threonine (Garfinkel et al. 1983). sgs-3 is also different from its two relatives in its high degree of glycosylation, with sugar molecules likely attached to threonine residues in the repetitive central module (Beckendorf and Kafatos 1976; Crowley et al. 1983).

Clones representing the chromosomal DNA of the $68 \mathrm{C}$ puff were collected by screening a genomic library with the cDNA clones and then with the newly obtained genomic clones. Overlapping clones containing over 50,000 bp of puff DNA were eventually characterized (Meyerowitz and Hogness 1982; Fig. 1). This DNA represents all of the sequences found in the $68 \mathrm{C}$ puff: If the leftmost fragment (the 2057 fragment in Fig. 1) from this chromosomal walk is biotinylated, hybridized in situ to surface-spread polytene chromosomes, then bound by anti-biotin antibody which is in turn bound with gold sphere-labeled anti-antibody, the gold spheres are seen in the transmission electron microscope to be positioned over the $68 \mathrm{C} 4$ band, which is just to the left of the puff. Similar experiments show that the right end of the $68 \mathrm{C}$ chromosomal walk (the 2058 fragment in Fig. 1) is in the $68 \mathrm{C} 7$ band, just to the right of the puff (Kress et al. 1985). The only region of the $68 \mathrm{C}$ puff that contains genes coding for abundant salivary gland RNAs is the central $5000 \mathrm{bp}$.

Hybridization and DNA sequencing studies show the structure of this region (Fig. 2). The $S g s 7$ and $S g s 8$ genes are transcribed as a divergent pair, with less than 500 bp separating their $5^{\prime}$ ends. Sgs 3 is transcribed from the same DNA strand as Sgs7, with almost $2000 \mathrm{bp}$ separating the poly(A) addition site of the Sgs7 RNA from the $5^{\prime}$ end of the $S g s 3$ gene. The $S g s 7$ and $S g s 3$ genes each have their own promoter, and their RNA products are not processed from a common precursor. Each of the genes has a single, small intervening sequence. The introns are in comparable positions in the three genes, all of them occurring between the second

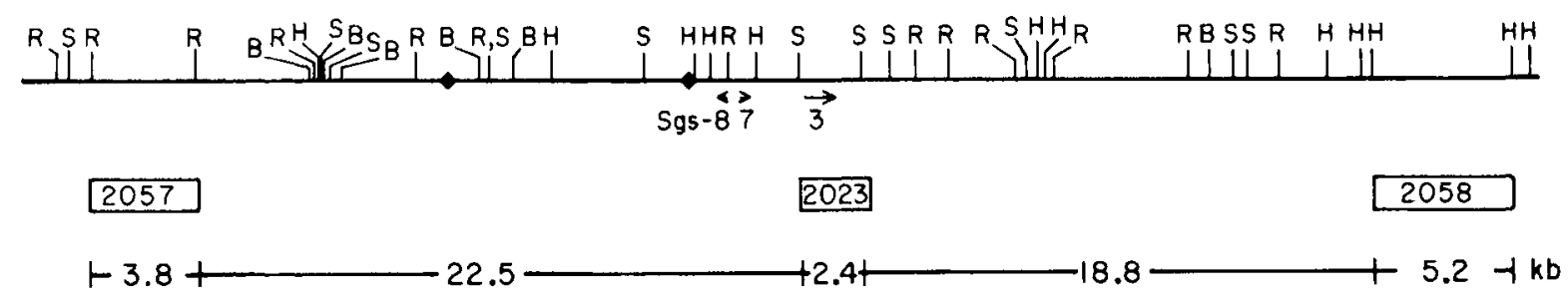

Figure 1. Restriction map of $68 \mathrm{C}$ puff DNA. The $68 \mathrm{C}$ puff is bounded on the left by the $3.8-\mathrm{kbp} 2057$ EcoRI fragment, and on the right by the $5.2-\mathrm{kbp} 2058$ HindIII fragment. The only genes in the puff DNA known to express in salivary glands are the Sgs3, Sgs7, and Sgs8 structural genes. The restriction endonuclease recognition sites shown are: R, EcoRI; S, Sall; B, BamHI; $\mathrm{H}$, HindIII. The diamonds on the map represent the termini of a transposable element of the roo family (Meyerowitz and Hogness 1982) that is present at this location in some strains, but not others. As drawn, the centromere is to the right and the tip of the left arm of the third chromosome to the left. 


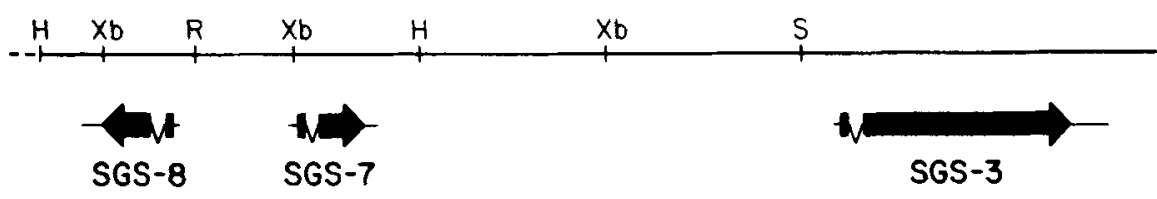

$1 \mathrm{~kb}$

Figure 2. Salivary gland transcripts at $68 \mathrm{C}$. The central portion of the $68 \mathrm{C}$ puff DNA contains the three $68 \mathrm{C}$ glue genes, transcribed in the directions shown. Translated portions of each glue RNA are shown as heavy lines, the upstream and downstream untranslated regions and the intervening sequences are shown as lighter lines. Above the transcripts is a restriction map of the genomic region containing the genes. The restriction endonuclease recognition sites shown are: $\mathrm{H}, H i n d I I l ; X b, X b a l ; R$, EcoRI; S, Sall; Xh, XhoI.

and third bases of the tenth codon. The Sgs8 intron is 69 nucleotides, the one in $S g s 766$ nucleotides, and the Sgs3 intervening sequence 73 bases long (Garfinkel et al. 1983). There is no detectable homology between the three introns, despite the similarity of the protein-coding portions of the mRNAs.

RNA hybridization experiments show that the three $68 \mathrm{C}$ glue RNAs are coordinately expressed. They are only found in third instar larvae, and only in salivary glands (Meyerowitz and Hogness 1982). Gel blots of RNA from salivary glands of individual midthird instar larvae show that any individual larva has either none or all three of the 68C RNAs present at any time; no individual (out of 25) had only one or two of the RNAs present (J. Kendall et al., unpubl.). Thus, the RNAs begin accumulating simultaneously. The three RNAs disappear together as well, just at the time of puparium formation. Therefore, in addition to the question of tissue- and time-specific regulation of each of the RNAs, there is a question of the mechanism of the precise coordination of expression of the three genes in the $68 \mathrm{C}$ cluster.

\section{Factors Required in trans for $68 \mathrm{C}$ Regulation}

Knowledge of the sequence organization of the $68 \mathrm{C}$ puff has allowed analysis of the sequences required in $c i s$, and the factors required in trans, for the normal regulation of the $68 \mathrm{C}$ genes. Two trans-acting factors are known. The first is ecdysterone, which not only causes puff regression, as described above, but is also required for the initiation of accumulation of the $68 \mathrm{C}$ RNAs, and for the later cessation of their synthesis.

The evidence that ecdysterone induces RNA accumulation at $68 \mathrm{C}$ comes largely from work with a temperature-sensitive $\mathrm{X}$-chromosomal mutation $l(l) s u$ $(f)^{15078}$. At $18^{\circ} \mathrm{C}$ flies homozygous or hemizygous for this mutation are normal. At $30^{\circ} \mathrm{C}$, they do not synthesize ecdysterone. If mutant larvae are raised to the restrictive temperature near the time of the second to third larval instar molt, they fail to accumulate any of the 68C RNAs in their salivary glands. If such larvae are then fed ecdysterone, accumulation of the RNAs begins (Hansson and Lambertsson 1983). Thus, the aspect of the $l(l) s u(f)^{\mathrm{s} 607 \mathrm{~g}}$ phenotype responsible for failure of $68 \mathrm{C}$ glue gene expression is the steroid, and the hormone is required for initiation of $68 \mathrm{C}$ gene expression. In these ecdysteroneless mutants, the $68 \mathrm{C}$ puff is present at the restrictive temperature, despite the absence of accumulation of the glue RNAs (Hansson et al. 1981)

Ecdysterone is also the signal that stops transcription of the three genes. If midthird instar salivary glands are dissected from larvae and cultured in a simple medium, they continue to synthesize the $68 \mathrm{C}$ glue RNAs for hours. This is demonstrated in pulse-labeling experiments, where salivary glands are cultured for 2 hours without label, then for 10 or 15 minutes in the presence of tritiated uridine or adenosine. RNA is then extracted from the glands and purified, and each of the individual $68 \mathrm{C}$ glue mRNAs is isolated from this mixture by hybrid selection (Crowley and Meyerowitz 1984). Scintillation spectrometry shows that each of the three RNAs is labeled in such an experiment, and thus is still being synthesized at high levels in the cultured glands. If ecdysterone is added to the culture medium to a concentration of $10^{-5} \mathrm{M}$ for as little as 15 minutes just before the pulse labeling, no significant radioactivity can be detected in the 68C RNAs after pulse-labeling and hybrid selection. Thus, the accumulation of newly synthesized $68 \mathrm{C}$ glue mRNA is very rapidly stopped by a short exposure to ecdysterone.

The hormonal control of the 68C RNAs in cultured salivary glands reflects the control mechanisms that operate in vivo. At about the time of the second to third instar molt, and again 5 hours before puparium formation, there are transient increases in the ecdysterone titer in the larval hemolymph (Berreur et al. 1979). The first of these ecdysterone peaks corresponds approximately to the time when $68 \mathrm{C}$ glue RNA begins accumulating, and the second corresponds to the time when the RNAs cease synthesis and when the $68 \mathrm{C}$ puff regresses. One might ask how the same hormone could both activate and inactivate the same genes in the same tissue. One possibility is that the initial hormone response of the salivary gland is to activate both the $68 \mathrm{C}$ 
genes and other genes, whose products eventually accumulate and cause a reversal in the cellular response to the hormone. Another possibility is that the levels of hormone involved in the two responses are different. These possibilities, and others, are currently being tested in our lab.

The second factor required in trans for initiation of expression of the three $68 \mathrm{C}$ glue RNAs is the product of a different $\mathrm{X}$-chromosomal gene, the $l(l) n p r-1$ gene. This mutation was first discovered as a late larval lethal that is not rescued by addition of ecdysterone to the larval medium (Kiss et al. 1978). It was afterward recognized that the $68 \mathrm{C}$ puff is present in late third instar in mutant larvae (Belyaeva et al. 1981), and demonstrated that in cultured salivary glands the $68 \mathrm{C}$ puff of these mutant larvae appears normal, but will not regress in response to the addition of exogenous ecdysterone (Crowley et al. 1984). Thus, it seemed possible that the 68C RNAs might be produced constitutively in the mutant. This is not the case. When $l(1) n p r-1$ mutant salivary gland RNA is subjected to electrophoresis and blotted to a nitrocellulose filter, and the filter-bound RNA hybridized with labeled 68C RNA-specific DNA probes, no $68 \mathrm{C}$ RNA is detected. Furthermore, if l(l)npr-l mutant salivary gland RNAs are pulse-labeled in organ culture experiments, no radioactivity is incorporated into the $68 \mathrm{C}$ glue RNAs, despite normal levels of incorporation into total salivary gland RNA (Crowley et al. 1984). Thus, the $I(1) n p r-1$ mutation prevents accumulation of newly synthesized RNA from the 68C puff, without affecting the appearance of the puff. Puffing and RNA accumulation are thus again shown to be separable. The $l(I) n p r-1$ mutation and a deletion of the $l(1) n p r-1$ locus both behave identically in their effect on 68C RNA levels, showing that the effect of the mutation is to eliminate the normal function of the locus (Crowley et al. 1984). It can therefore be concluded that the normal product of the $l(l) n p r-l$ locus is required in trans for initiation of expression of the $68 \mathrm{C}$ glue genes.

What is the $1(1) n p r-1$ product? One possibility is that it is a component of the ecdysterone receptor, which binds ecdysterone when it enters cells. This binding is believed to be the first step in the action of the hormone. One piece of evidence that indicates that the gene product may not be a crucial component of the receptor is that the $l(1) n p r-1$ mutation only reduces, and does not eliminate, accumulation of RNA from the $3 \mathrm{C}$ glue puff, which codes for the sgs-4 polypeptide (Crowley et al. 1984). This RNA, like the 68C RNAs, requires ecdysterone to trigger the initiation of its accumulation (Hansson and Lambertsson 1983). Thus, if the l(l)npr1 gene product is a component of the ecdysterone receptor, it is a component that is only required for some, and not other, ecdysterone responses.

\section{Sequences Required in cis for $68 \mathrm{C}$ Regulation}

What DNA or RNA sequences might the trans-acting factors interact with? Classical genetic experiments show that no more than 20,000 bp of contiguous DNA sequence is required for normal puffing and expression of the $68 \mathrm{C}$ glue genes: $D f(3 L) v i^{3}$ is a deficiency that removes a large chromosomal segment normally found to the right of the $68 \mathrm{C}$ puff. The left deficiency breakpoint is just over $1000 \mathrm{bp}$ downstream of the $3^{\prime}$ end of the Sgs 3 gene. This deficiency has no effect on expression of the adjacent $S g s 3$ gene or on the puffing of the 68C DNA. In $(3 L) H R 15$ is an inversion of a chromosomal region that includes a substantial part of the left arm of the third chromosome. The right inversion breakpoint is about $15 \mathrm{kbp}$ to the left of the $3^{\prime}$ end of $S g s 8$, and the effect of the inversion is to move sequences normally found adjacent to the $68 \mathrm{C}$ puff to a distant location. This inversion has no effect on expression of any of the $68 \mathrm{C}$ glue genes or on $68 \mathrm{C}$ puffing (M.A. Crosby and E.M. Meyerowitz, in prep.).

Thus, the sequences that control the $68 \mathrm{C}$ glue genes are near the genes. These classical experiments do not show whether there is only one set of sequences that regulate all three of the genes, thus explaining why these coordinately regulated genes may be found in a gene cluster, or whether there are separate regulatory regions for each of the genes. The DNA sequence of the genes is no help in this respect either: There are no recognizable stretches of similar sequences either upstream or downstream of the three genes that are obvious candidates for control sequences (Garfinkel et al. 1983). To localize the regulatory sequences that interact with trans-acting regulatory factors, P-element transformation experiments have been performed. In these experiments various fragments of the 68C DNA have been cloned into P-element transformation vectors (Rubin and Spradling 1982; Goldberg et al. 1983; Spradling and Rubin 1983) adjacent to genes whose product is selectable or easily recognized in adult flies (alcohol dehydrogenase, which confers resistance to high levels of ethanol, or xanthine dehydrogenase, which affects fly eye color). These constructs are then injected into Drosophila embryos in a way that allows reintegration of the cloned genes into random locations in the chromosomes of the primordial germ cells of the embryos. Transformed lines are recognized by the phenotype that results from expression of the selected marker, and the expression of the unselected $68 \mathrm{C}$ fragments is assayed in the salivary glands of larvae from these lines.

Most of the experiments have been aimed at determining the extent of $S g s 3$ regulatory sequences. To assay expression of introduced Sgs3 genes, it is necessary to distinguish the RNA and protein products of the introduced genes from those of endogenous genes. This has been done in our laboratory by injecting genes from the Oregon-R wild-type strain into embryos whose Sgs3 genes derived either from the Hikone-R or Formosa wild strains. While the Sgs3 RNA in the Oregon-R strain is 1120 nucleotides in length, those from the other strains are smaller, and are easily separated from the Oregon-R type by agarose gel electrophoresis. If an Sgs3 gene is reintroduced to the fly genome, along with 
$2.27 \mathrm{~kb}$ of upstream sequence and some downstream flanking sequence as well, it expresses RNA normally: The RNA is only found in third larval instar and only in salivary glands. It accumulates to an approximately normal level, and is translated to a normal amount of sgs-3 protein (M.A. Crosby and E.M. Meyerowitz, in prep.). The fact that sgs-3 protein is made demonstrates that RNA from the introduced gene is spliced, as failure of splicing would lead to an RNA that would translate to a short peptide easily distinguished from sgs-3. Despite the proper expression of RNA and protein from the introduced gene, in at least one case where careful measurements have been made, there is no puff at the site of integration of the new gene. That an Sgs3 gene with $2.7 \mathrm{~kb}$ of upstream sequence expresses RNA normally without puffing has also been shown by Richards and his collaborators, in experiments in which the Sgs3 gene of the Formosa strain gene has been integrated into the genome of a strain that produces a 1120-nucleotide Sgs3 RNA (Richards et al. 1983.) If only 130 nucleotides of sequence are left upstream of the Sgs3 gene, our laboratory and Richards' obtain somewhat different results. We find, in experiments using an alcohol dehydrogenase vector and involving introduction of an Oregon- $R$ gene into a Formosa background, that the introduced genes express at the correct time and in the correct tissue, but at a level 10to 40 -fold lower than normal (M.A. Crosby and E.M. Meyerowitz, in prep.). They find that a Formosa $S g s 3$ gene introduced with a xanthine dehydrogenase vector into a strain with an Oregon-R-sized endogenous gene gives a level of RNA more than 400 -fold lower than normal (Bourouis and Richards 1985). The basis of difference between the results of the two labs is not yet known; differences in transformation vector, genetic background of transformed flies, and strain from which the introduced gene derived are all possibilities. Since the Richards lab has transformants with 1.0 and $1.5 \mathrm{~kb}$ of upstream $S g s 3$ sequence that express the RNA at a reduced level, but in appropriate tissue and stage, all of the work agrees in the conclusion that the upstream regulatory sequences of the $S g s 3$ gene contain two separable elements. One is responsible for the level of expression of the RNAs and includes sequences from -130 to -2270 from the start of transcription; the other controls the tissue and time of expression and is closer to or in the gene.

The $S g s 3$ experiments demonstrate that this gene has its own regulatory sequences nearby but do not reveal if the $\mathrm{Sgs} 7$ and $\mathrm{Sgs} 8$ genes respond to the same sequences as the $S g s 3$ gene, or have their own regulatory regions. To find out, we have fused the $S g s \&$ gene to an Escherichia coli $\beta$-galactosidase gene, creating a fusion gene whose RNA and protein products are easily distinguished from the products of the endogenous Sgs8 genes. This construct, with upstream sequences derived from the $S g s 8$ gene, and an $S g s 83^{\prime}$ untranslated region and poly(A) addition site added downstream of the $\beta$ galactosidase gene, has been cloned into a xanthine dehydrogenase P-element vector. Larvae homozygous for the newly introduced construct have been tested both for the fusion RNA and for $E$. coli $\beta$-galactosidase activity. Both the RNA and the activity are present in third instar larval salivary glands, and in no other third instar tissues (E.M. Meyerowitz, unpubl.). Since this construct shares no 68C sequences with the $S g s 3$ constructs, the $S g s 8$ gene must have its own regulatory sequences, and need not be near an $S g s 3$ gene to express appropriately. Therefore we can exclude models that explain coordinate expression of the $68 \mathrm{C}$ genes by postulating that all three of the genes rely on the same regulatory sequences. We are also led to the conclusion that the clustering of the three genes at $68 \mathrm{C}$ is a consequence of their evolution by local duplication followed by divergence, and not a functional necessity.

\section{Interaction of trans-acting Factors and cis-acting Sequences}

By crossing the trans-acting mutations into strains containing introduced modified glue genes, we can learn about the sequences that interact with the transacting factors, and about the factors themselves. An example of such an experiment is one in which the l(1)npr-1 mutation was crossed into a transformed strain bearing the Hikone-R $S g s 3$ variant as its endogenous $S g s 3$ genes and, closer to the tip of the left arm of the third chromosome, an introduced Sgs 3 construct with $2.27 \mathrm{~kb}$ of upstream sequence, the Oregon- $\mathrm{R}$ variant of the $S g s 3$ gene, and approximately $2.5 \mathrm{~kb}$ of downstream sequence. In the presence of a wild-type l(I)npr-I locus, this introduced gene expresses Sgs3 RNA and protein at approximately normal levels and in the appropriate developmental stage and tissue. When this wild-type locus is replaced with the l(l)npr1 mutation, neither the endogenous nor the introduced genes express (Crowley et al. 1984). Thus, the normal product of the l(l)npr-l locus, or something induced by the product of this locus, interacts with sequences in or near the $S g s 3$ gene to allow its expression. The sequences could be DNA upstream, in, or downstream of the gene, or sequences of the RNA transcript of the gene. Just which sequences are involved is being determined with constructs that separate upstream, genic, and downstream sequences. Additional experiments may reveal whether the product of the wild-type l(I)npr1 gene is a factor that directly binds to DNA or RNA and allows its expression, or is a product that causes a repressor to leave the DNA or RNA. If the former is true, constructs that express Sgs3 normally will always fail to express when the $1(1)$ npr-I mutation is present. If the latter is the case, some constructs that lack the repressor binding site may express the $S g s 3$ gene despite the absence of the wild-type l(l)npr-l product.

Similar experiments with strains containing introduced $S g s 8 \beta$-galactosidase fusion genes show that the l(1)npr-1 product acts, either directly or indirectly, on sequences in or near the Sgs8 gene in addition to se- 
quences near the $S g s 3$ gene. When the $l(1) n p r-1$ mutation is crossed into one of the strains carrying the fusion construct, the fusion gene no longer directs synthesis of $\beta$-galactosidase in third instar larvae (K. VijayRaghavan and E.M. Meyerowitz, unpubl.). The l(1)npr-1 product thus acts independently on the Sgs3 and $S g s 8$ genes.

One further approach to identifying the $68 \mathrm{C}$ glue puff sequences recognized by trans-acting factors involves analysis of regions of the genomes of other Drosophila species that are homologous with the $D$. melanogaster $68 \mathrm{C}$ puff. It is known that several species of the melanogaster species subgroup of Drosophila have puffs at their equivalent of polytene chromosome location 68C (Ashburner and Lemeunier 1972; Ashburner and Berendes 1978). These regions have been cloned and characterized (Meyerowitz and Martin 1984). All of them contain genes homologous with the $68 \mathrm{C}$ glue genes of $D$. melanogaster that exist in coordinately regulated clusters and express abundant polyadenylated RNAs in third instar salivary glands. D. simulans and $D$. erecta both have three genes in the $68 \mathrm{C}$-homologous clusters, with two small genes that cross-hybridize with $S g s 8$ or $S g s 7$, and one large gene that has homology with Sgs3. D. yakuba and D. teissieri have four genes, three small genes, and one Sgs3-like large gene. Examination of the DNA sequences of these genes is the converse of a mutagenesis experiment. In a mutagenesis experiment, one seeks the smallest changes in a DNA sequence that alter its function. In these experiments, we are looking at the largest amount of divergence in a DNA sequence that leaves its function intact. The sequences that have not diverged are expected to be those whose function is critical, including those that interact with diffusible regulatory factors. Those sequences that are bound by regulatory factors are expected to diverge more slowly than others, because a change in an important base in such a sequence would have to be accompanied by a corresponding change in the regulatory factor if function is to be retained. We are currently analyzing sequences of the Sgs3-equivalent genes, and their flanking regions, from $D$. simulans, $D$. erecta, and D. yakuba. There are conserved sequences upstream of the genes, separated by regions of low sequence homology (C.H. Martin and E.M. Meyerowitz, unpubl.). When the conserved regions have been thoroughly analyzed, interspecies transformation experiments will allow us to determine if these sequences do indeed serve the same function in all of the species. In addition, the $S g s 3$ gene sequences will enable us to see how the repetitive central protein module of the gene evolves, and perhaps to understand its origin.

\section{Conclusions and Further Questions}

The Drosophila $68 \mathrm{C}$ glue gene cluster is a chromosomal region whose transcription is regulated hormonally and in a tissue-specific manner. In wild-type larvae transcription of the genes of the cluster and visible chromatin decondensation are coincident. Analysis of trans-acting mutations has revealed two diffusible factors required by the $68 \mathrm{C}$ genes for proper RNA expression, and has allowed a rough localization of the sequences with which one of them interacts. In vitro mutagenesis of the $\mathrm{Sgs} 3$ gene has shown that the sequences required in cis for normal gene activity contain two functional regions, one controlling the level of gene transcription and one directing the time and tissue of gene expression. The $S g s 8$ gene has been demonstrated to have its own regulatory sequences, independent of the similar Sgs3 sequences. Nonetheless, many questions remain. The identity of the molecules that interact with the $68 \mathrm{C}$ sequences is unknown, as is their exact site of interaction, and their precise effects. A major question raised by our work, and not yet answered, regards the relation of gene expression and puffing at $68 \mathrm{C}$. Puffing in wild-type salivary glands always corresponds with high-level transcription of the puffed DNA, implying that puffing is either a precondition to high-level gene expression, or a result of it. In mutant and transformed larvae, however, puffing and high-level expression of the $68 \mathrm{C}$ glue mRNAs have been separated in several ways. The puff can be present and normal in appearance without concomitant accumulation of the puff RNAs, as in $l(1) s u(f)^{15678}$ and $l(1) n p r-1$ larvae, and at least one of the $68 \mathrm{C}$ genes ( $\mathrm{Sgs} 3$, which accounts for more than half of the transcription in the $68 \mathrm{C}$ gene cluster) can express at an approximately normal level without giving rise to a recognizable puff. In these abnormal situations, puffing is neither necessary nor sufficient for abundant transcription, and highlevel transcription is neither necessary nor sufficient for puffing. Additional experiments designed to reveal the relation of gene transcription and polytene chromosome puffing, and to define better the trans-acting factors and cis-acting sequences that control the $68 \mathrm{C}$ glue genes, are in progress.

\section{ACKNOWLEDGMENTS}

Our $68 \mathrm{C}$ puff work has been funded by National Institutes of Health grant GM28075 to E.M.M., M.D.G., and P.H.M. are supported by National Research Service Award T32 GM07616; C.H.M. is supported by a National Science Foundation predoctoral fellowship; and K.V. is supported by a Procter and Gamble postdoctoral fellowship.

\section{REFERENCES}

AKam, M.E., D.B. RoberTs, G.P. Richards, and M. AshBURNER. 1978. Drosophila: The genetics of two major larval proteins. Cell 13: 215.

Ashburner, M. 1967. Patterns of puffing activity in the salivary gland chromosomes of Drosophila. I. Autosomal puffing patterns in a laboratory stock of Drosophila melanogaster. Chromosoma 21: 398.

-1973. Sequential gene activation by ecdysone in polytene chromosomes of Drosophila melanogaster. I. Dependence upon ecdysone concentration. Dev. Biol. 35: 47.

-1974. Sequential gene activation by ecdysone in poly- 
tene chromosomes of Drosophila melanogaster. II. The effects of inhibitors of protein synthesis. Dev. Biol. 39: 141 .

Ashburner, M. and H.D. Berendes. 1978. Puffing of polytene chromosomes. In The genetics and biology of Drosophila (ed. M. Ashburner and T.R.F. Wright), vol. 2B, p. 315. Academic Press, London.

AshburNer, M. and F. Lemeunier. 1972. Patterns of puffing activity in the salivary gland chromosomes of Drosophila. VIl. Homology of puffing patterns on chromosome arm $3 \mathrm{~L}$ in $D$. melanogaster and $D$. yakuba, with notes on puffing in D. teissieri. Chromosoma 38: 283.

BECKENDORF, S.K. and F.C. KAFATOS. 1976. Differentiation in the salivary glands of Drosophila melanogaster: Characterization of the glue proteins and their developmental appearance. Cell 9: 365.

Belyaeva, E.S., I.E. Vlassova, Z.M. Biyasheva, V.T. KaKPAKOV, G. RICHARDS, and I.F. ZhIMULEV. 1981. Cytogenetic analysis of the 2B3-4-2B11 region of the X-chromosome of Drosophila melanogaster. II. Changes in $20-\mathrm{OH}$ ecdysone puffing caused by genetic defects of puff 2B5. Chromosoma 84: 207.

BERENDES, H.D. and M. AShbuRner. 1978. The salivary glands. In The genetics and biology of Drosophila (ed. M. Ashburner and T.R.F. Wright), vol. 2B, p. 453. Academic Press, London.

Berreur, P., P. Porcheron, J. Berreur-Bonnefant, and P. SIMPSON. 1979. Ecdysteroid levels and pupariation in Drosophila melanogaster. J. Exp. Zool. 201: 347.

Bourouis, M. and G. RichardS. 1985. Remote regulatory sequences of the Drosophila glue gene $S g s-3$ as revealed by P element transformation. Cell 40: 349.

BRIDGes, C.B. 1935. Salivary chromosome maps. J. Hered. 26: 60 .

Crowley, T.E. and E.M. MeYerowitz. 1984. Steroid regulation of RNAs transcribed from the Drosophila $68 \mathrm{C}$ polytene chromosome puff. Dev. Biol. 102: 110.

Crowley, T.E., M.W. Bond, and E.M. MEYerowitz. 1983. The structural genes for three Drosophila glue proteins reside at a single polytene chromosome puff locus. Mol. Cell. Biol. 3: 623.

Crowley, T.E., P.H. Mathers, and E.M. Meyerowitz. 1984. A trans-acting regulatory product necessary for expression of the Drosophila melanogaster $68 \mathrm{C}$ glue gene cluster. Cell 39: 149.

FraENKEL, G. and V.J. Brookes. 1953. The process by which the puparia of many species of flies become fixed to a substrate. Biol. Bull. 105: 442.

Garfinkel, M.D., R.E. PruitT, and E.M. Meyerowitz. 1983. DNA sequences, gene regulation and modular protein evolution in the Drosophila $68 \mathrm{C}$ glue gene cluster. J. $\mathrm{Mol}$. Biol. 168: 765 .

GaUTAM, N. 1983. Identification of two closely located larval salivary protein genes in Drosophila melanogaster. Mol. Gen. Genet. 189: 495.

Goldberg, D.A., J.W. Posakony, and T. Maniatis. 1983. Correct developmental expression of a cloned alcohol de- hydrogenase gene transduced into the Drosophila germ line. Cell 34: 59.

GulLD, G.M. and E.M. SHORE. 1984. Larval salivary gland secretion proteins in Drosophila: Identification and characterization of the Sgs-5 structural gene. J. Mol. Biol. 179: 289.

HANSSON, L. and A. LAMBERTSSON. 1983. The role of su(f gene function and ecdysterone in transcription of glue polypeptide mRNAs in Drosophila melanogaster. Mol. Gen. Genet. 192: 395.

HANSSON, L., K. LiNerUTH, and A. LAMBERTSSON. 1981. Effects of the $l(1) s u(f)^{15678}$ mutation of Drosophila melanogaster on glue protein synthesis. Wilhelm Roux's Arch. Dev. Biol. 190: 308.

KIss, I., J. Szabad, and J. MajoR. 1978. Genetic and developmental analysis of puparium formation in Drosophila. Mol. Gen. Genet. 164: 77.

KORGE, G. 1975. Chromosome puff activity and protein synthesis in larval salivary glands of Drosophila melanogaster. Proc. Natl. Acad. Sci. 72: 4550.

- 1977. Larval saliva in Drosophila melanogaster: Production, composition and relation to chromosome puffs Dev. Biol. 58: 339.

Kress, H., E.M. Meyerowitz, and N. DavidSon. 1985. High resolution mapping of in situ hybridized biotinylated DNA to surface spread Drosophila polytene chromosomes. Chromosoma (in press).

MEYerowitz, E.M. and D.S. Hogness. 1982. Molecular organization of a Drosophila puff site that responds to ecdysone. Cell 28: 165.

Meyerowitz, E.M. and C.H. MarTin. 1984. Adjacent chromosomal regions can evolve at very different rates: Evolution of the Drosophila $68 \mathrm{C}$ glue gene cluster. J. $\mathrm{Mol}$. Evol. 20: 251

MUSKAVITCH, M.A.T. and D.S. HoGNESS. 1980. Molecular analysis of a gene in a developmentally regulated puff of Drosophila melanogaster. Proc. Natl. Acad. Sci. 77: 7362.

Richards, G., A. CASSAB, M. Bourouis, B. JaRry, and C. Dissous. 1983. The normal developmental regulation of a cloned Sgs-3 "glue" gene chromosomally integrated in Drosophila melanogaster by $\mathrm{P}$ element transformation. $E M B O$ J. 2: 2137.

Rubin, G.M. and A.C. SPRADLing. 1982. Genetic transformation of Drosophila with transposable element vectors. Science 218: 348 .

SPRADLING, A.C. and G.M. RuBiN. 1983. The effect of chromosomal position on the expression of the Drosophila xanthine dehydrogenase gene. Cell 34: 47.

Velissariou, V. and M. AshbuRner. 1980. The secretory proteins of the larval salivary glands of Drosophila melanogaster. Chromosoma 77: 13.

- 1981. Cytogenetic and genetic mapping of a salivary gland secretion protein in Drosophila melanogaster. Chromosoma 84: 173 .

WOLFNER, M. 1980. "Ecdysone-responsive genes of the salivary gland of Drosophila melanogaster." Ph.D. thesis, Stanford University, Stanford, California. 